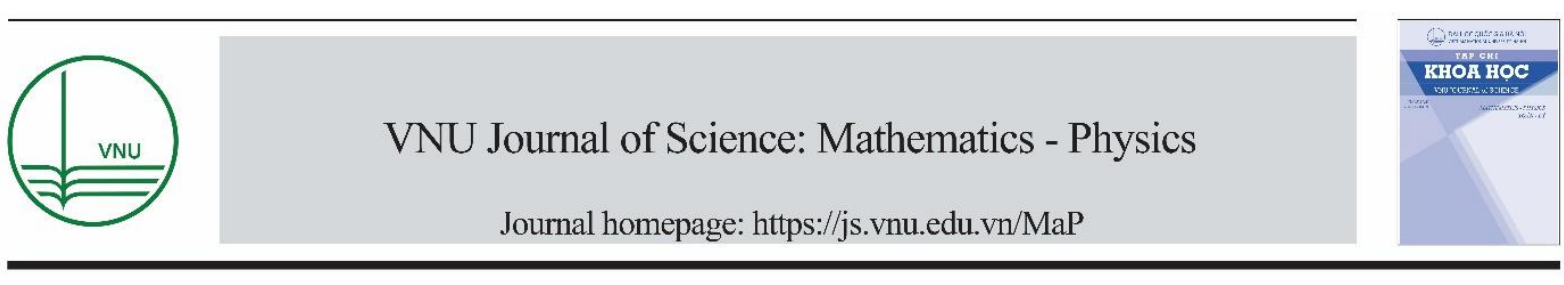

Original Article

\title{
Magneto-thermoelectric Effects in Cylindrical Quantum Wire under the Influence of Electromagnetic Wave for Electron-optical Phonon Scattering
}

\author{
Nguyen Quang Bau, Doan Minh Quang, Tran Hai Hung* \\ Faculty of Physics, VNU University of Science, 334 Nguyen Trai, Hanoi, Vietnam
}

Received 03 October 2019

Revised 10 December 2019; Accepted 16 December 2019

\begin{abstract}
This paper studies the influence of a strong electromagnetic wave (EMW) on the magneto-thermoelectric effects in a cylindrical quantum wire with an infinite potential (CQWIP) (for electron - optical phonon scattering) under the influence of electric field $\boldsymbol{E}_{\mathbf{1}}=\left(0,0, E_{1}\right)$, magnetic field $\boldsymbol{B}=(0, B, 0)$ and a strong EMW (laser radiation) $\boldsymbol{E}=\left(0,0, E_{0} \sin \Omega t\right.$ ) (where $E_{0}$ and $\Omega$ are amplitude and frequency of EMW, respectively), based on the quantum kinetic equation for electrons. The study obtained the analytic expressions for the kinetic tensors $\sigma_{i k}, \beta_{i k}, \gamma_{i k}, \xi_{i k}$ and the Ettingshausen coefficient (EC) in the CQWIP with the dependence on the frequency, the amplitude of EMW, the Quantum Wire (CQWIP) parameters, the magnetic field and the temperature. The study results were numerically evaluated and graphed for GaAs/GaAsAl quantum wire. Then, the results in this case were compared with those in the case of the bulk semiconductors and other lowdimension systems in order to show the difference and the novelty of the current results. Moreover, it is realized that as the EMW frequency increases, the EC fluctuates with a stable trend, and the appearance of the Shubnikov-de Haas ( $\mathrm{SdH}$ ) oscillations pattern when the dependence of EC on the magnetic field is surveyed.
\end{abstract}

Keywords: Quantum wire, Ettingshausen effect, electron-optical phonons cattering, quantum kinetic equation

\section{Introduction}

In recent years, the semiconductor materials have been widely used in electronics. The development of semiconductor electronics is mainly based on the phenomenon of contact $p-n$ and the doped ability to alter the physical properties of crystals. The particle motion is limited along specific

\footnotetext{
${ }^{*}$ Corresponding author.

Email address: haihung307@gmail.com
}

https//doi.org/ 10.25073/2588-1124/vnumap.4400 
coordinates within a tiny area of less than hundreds $\AA$. If the size of the area is comparable with Debroglie wavelength of the particle, the energy spectrum and wave function will be quantized. Then, the size effect appeared and makes almost physical properties of material change [1]. The properties of low-dimension system such as: Hall effect [2-4], absorption of electromagnetic waves, relative magnetoresistance, etc [5-8] are very different from the previous work studied on bulk semiconductor [9-12]. The magneto-thermoelectric effect, often called as Ettingshausen effect, which has just been researched in bulk semiconductors [13-16] is one of the electrical, magnetic and thermal effects of semiconductor systems. Furthermore, the magneto-thermoelectric effect in low-dimension systems has just been studied in two-dimension system (quantum well) [17-19]. Due to the fact that the electron's energy spectrum and single wave function in two-dimension systems are different from the case of one-dimension systems, the Ettingshausen effect characterizes in each case lead us to separated results. The Ettingshausen effect has also been researched on the rectangular quantum wire by using the quantum method (quantum kinetic equation). However, this quantum effect has not been studied in cylindrical quantum wire with different cases of confined potential.

So, in order to accomplish the Ettingshausen quantum theory in low-dimension semiconductor systems, we use the quantum kinetic equation to calculate the EC in CQWIP, which is used a lot in studies about one-dimension system, under the influence of electromagnetic wave. We have discovered some differences between the results obtained in this case and those in the case of the bulk semiconductors. Numerical calculations are carried out with a specifically cylindrical quantum wire GaAs/GaAs:Al. To the limitation in others low-dimension system, we obtained the results close to previous studies. Then, when surveying the EC in some parameters, we explored the new which is only acquired in our work.

\section{Calculation of Ettingshausen coefficient in cylindrical quantum wire in the presence of electromagnetic wave}

In this report, we consider a cylindrical quantum wire of the normalization radius $R$ with the infinite confined potential $(\mathrm{V}(\overrightarrow{\mathrm{r}})=0, r \leq R$ and $\mathrm{V}(\vec{r})=\infty, \mathrm{r}>\mathrm{R})$ elsewhere subjected to a crossed electric field $\boldsymbol{E}_{\mathbf{1}}=\left(0,0, E_{1}\right)$ and magnetic field $\boldsymbol{B}=(0, B, 0)$ in the presence of a strong EMW (laser radiation) characterized by electric field $\boldsymbol{E}=\left(0,0, E_{0} \sin \Omega t\right)$.

$$
\begin{aligned}
& \psi_{n, l, \overrightarrow{p_{z}}}(r, \phi, z)=\left\{\begin{array}{cc}
\sqrt{\frac{1}{V_{0}} e^{i m \phi} e^{i \overrightarrow{p_{z}} z} \psi_{n, l}(r),}, \quad r \leq R \\
0 \quad, \quad r>R
\end{array}\right. \\
& \varepsilon_{n, l, \overrightarrow{p_{z}}}=\frac{\hbar^{2}{\overrightarrow{p_{z}}}^{2}}{2 m^{*}}+\hbar \omega_{c}\left(N+\frac{n}{2}+\frac{l}{2}+\frac{1}{2}\right)-\frac{1}{2 m^{*}}\left(\frac{e E_{1}}{\omega_{c}}\right)^{2} ; n, l, N=0,1,2, \ldots
\end{aligned}
$$

Here: $\overrightarrow{p_{z}}$ is the wave vector in the z-direction, $\omega_{c}$ is the cyclotron frequency and $\psi_{n, l}(r)=$ $\frac{1}{J_{n+1}\left(A_{n, l}\right)} J_{n}\left(A_{n, l} \frac{r}{R}\right)$ is the diametral wave function.

After using the Hamiltonian of the system of confened electron - optical phonon in a CQW in the second quantization presentation in $\mathrm{E}_{1}, \mathrm{~B}, \mathrm{E}$, we obtain the quantum kinetic equation for distribution function of electron, then we calculate the curent density and thermal flux density formula, we obtain the kinetic tensor $\sigma_{i k}, \beta_{i k}, \gamma_{i k}, \xi_{i k}$. We can have the expression of the EC:

$$
P=\frac{1}{H} \frac{\sigma_{x x} \gamma_{x y}-\sigma_{x y} \gamma_{x x}}{\sigma_{x x}\left[\beta_{x x} \gamma_{x x}-\sigma_{x x}\left(\xi_{x x}-K_{L}\right)\right]}
$$


Where: $\quad \sigma_{i j}=\frac{e^{2} L R^{2} \hbar^{4}}{8 \pi^{2} m^{* 3}} \frac{\tau\left(\varepsilon_{F}\right)}{1+\omega_{c}^{2} \tau\left(\varepsilon_{F}\right)^{2}} \sum_{n, l} \Delta_{Q}^{\frac{2}{3}}\left[\delta_{i k}+\omega_{c} \tau\left(\varepsilon_{F}\right) \varepsilon_{i k j}+\omega_{c}^{2} \tau\left(\varepsilon_{F}\right)^{2} h_{i} h_{k}\right]+\frac{e^{3} \hbar^{5} \omega_{0} L}{48 m^{3} \pi \kappa}\left(\frac{1}{\chi_{\infty}}-\right.$ $\left.\frac{1}{\chi_{0}}\right) \sum_{n, l, n^{\prime}, l^{\prime}} \frac{I_{n, l, n^{\prime}, l^{\prime}}{ }^{\hbar}}{e^{\hbar \omega_{0} / k_{B} T}-1}\left\{(S H 1+S H 2) \frac{\tau\left(\varepsilon_{F}-\hbar \omega_{0}\right)}{1+\omega_{c}^{2} \tau\left(\varepsilon_{F}-\hbar \omega_{0}\right)^{2}}\left[\delta_{i k}+\omega_{c} \tau\left(\varepsilon_{F}-\hbar \omega_{0}\right) \varepsilon_{i k j}+\omega_{c}{ }^{2} \tau\left(\varepsilon_{F}-\right.\right.\right.$ $\left.\left.\hbar \omega_{0}\right)^{2} h_{i} h_{k}\right]+(S H 3+S H 4) \frac{\tau\left(\varepsilon_{F}+\hbar \omega_{0}\right)}{1+\omega_{c}{ }^{2} \tau\left(\varepsilon_{F}+\hbar \omega_{0}\right)^{2}}\left[\delta_{i k}+\omega_{c} \tau\left(\varepsilon_{F}+\hbar \omega_{0}\right) \varepsilon_{i k j}+\omega_{c}{ }^{2} \tau\left(\varepsilon_{F}+\right.\right.$ $\left.\left.\left.\hbar \omega_{0}\right)^{2} h_{i} h_{k}\right]\right\}+\frac{e^{5} \hbar^{5} \omega_{0} L E_{o}^{2}}{192 m^{5} \Omega^{4} \pi \kappa}\left(\frac{1}{\chi_{\infty}}-\frac{1}{\chi_{0}}\right) \sum_{n, l, n^{\prime}, l^{\prime}} \frac{I_{n, l, n^{\prime}, l^{\prime}} e^{\hbar \omega_{0} / k_{B} T}}{19 H 5 \frac{\tau\left(\varepsilon_{F}-\hbar \omega_{0}+\hbar \Omega\right)}{1+\omega_{c}^{2} \tau\left(\varepsilon_{F}-\hbar \omega_{0}+\hbar \Omega\right)^{2}}}\left[\delta_{i k}+\right.$ $\left.\omega_{c} \tau\left(\varepsilon_{F}-\hbar \omega_{0}+\hbar \Omega\right) \varepsilon_{i k j}+\omega_{c}^{2} \tau\left(\varepsilon_{F}-\hbar \omega_{0}+\hbar \Omega\right)^{2} h_{i} h_{k}\right]+S H 6 \frac{\tau\left(\varepsilon_{F}-\hbar \omega_{0}-\hbar \Omega\right)}{1+\omega_{c}^{2} \tau\left(\varepsilon_{F}-\hbar \omega_{0}-\hbar \Omega\right)^{2}}\left[\delta_{i k}+\right.$ $\left.\omega_{c} \tau\left(\varepsilon_{F}-\hbar \omega_{0}-\hbar \Omega\right) \varepsilon_{i k j}+\omega_{c}^{2} \tau\left(\varepsilon_{F}-\hbar \omega_{0}-\hbar \Omega\right)^{2} h_{i} h_{k}\right]+S H 7 \frac{\tau\left(\varepsilon_{F}+\hbar \omega_{0}+\hbar \Omega\right)}{1+\omega_{c}^{2} \tau\left(\varepsilon_{F}+\hbar \omega_{0}+\hbar \Omega\right)^{2}}\left[\delta_{i k}+\right.$ $\left.\omega_{c} \tau\left(\varepsilon_{F}+\hbar \omega_{0}+\hbar \Omega\right) \varepsilon_{i k j}+\omega_{c}{ }^{2} \tau\left(\varepsilon_{F}+\hbar \omega_{0}+\hbar \Omega\right)^{2} h_{i} h_{k}\right]+S H 8 \frac{\tau\left(\varepsilon_{F}+\hbar \omega_{0}-\hbar \Omega\right)}{1+\omega_{c}{ }^{2} \tau\left(\varepsilon_{F}+\hbar \omega_{0}-\hbar \Omega\right)^{2}}\left[\delta_{i k}+\right.$ $\left.\left.\omega_{c} \tau\left(\varepsilon_{F}+\hbar \omega_{0}-\hbar \Omega\right) \varepsilon_{i k j}+\omega_{c}^{2} \tau\left(\varepsilon_{F}+\hbar \omega_{0}-\hbar \Omega\right)^{2} h_{i} h_{k}\right]\right\}$

$\beta_{i j}=\frac{e^{3} \hbar^{5} \omega_{0} L}{48 m^{3} \pi \kappa}\left(\frac{1}{\chi_{\infty}}-\frac{1}{\chi_{0}}\right) \sum_{n, l, n^{\prime}, l^{\prime}} \frac{I_{n, l, n^{\prime}, l^{\prime}} e^{\hbar \omega_{0} / k_{B} T}-1}{\hbar \frac{\hbar \omega_{0}}{T}}(S H 1+S H 2) \frac{\tau\left(\varepsilon_{F}-\hbar \omega_{0}\right)}{1+\omega_{c}^{2} \tau\left(\varepsilon_{F}-\hbar \omega_{0}\right)^{2}}\left[\delta_{i k}+\right.$ $\left.\omega_{c} \tau\left(\varepsilon_{F}-\hbar \omega_{0}\right) \varepsilon_{i k j}+\omega_{c}^{2} \tau\left(\varepsilon_{F}-\hbar \omega_{0}\right)^{2} h_{i} h_{k}\right]-\frac{\hbar \omega_{0}}{T}(S H 3+S H 4) \frac{\tau\left(\varepsilon_{F}+\hbar \omega_{0}\right)}{1+\omega_{c}{ }^{2} \tau\left(\varepsilon_{F}+\hbar \omega_{0}\right)^{2}}\left[\delta_{i k}+\right.$ $\left.\left.\omega_{c} \tau\left(\varepsilon_{F}+\hbar \omega_{0}\right) \varepsilon_{i k j}+\omega_{c}^{2} \tau\left(\varepsilon_{F}+\hbar \omega_{0}\right)^{2} h_{i} h_{k}\right]\right\}+\frac{e^{5} \hbar^{5} \omega_{0} L E_{o}^{2}}{192 m^{5} \Omega^{4} \pi \kappa}\left(\frac{1}{\chi_{\infty}}-\right.$ $\left.\frac{1}{\chi_{0}}\right) \sum_{n, l, n^{\prime}, l^{\prime}} \frac{I_{n, l, n^{\prime}, l^{\prime}}{ }^{\hbar \omega_{0} / k_{B} T}}{e^{2}-1}\left\{\frac{\hbar \omega_{0}-\hbar \Omega}{T} S H 5 \frac{\tau\left(\varepsilon_{F}-\hbar \omega_{0}+\hbar \Omega\right)}{1+\omega_{c}^{2} \tau\left(\varepsilon_{F}-\hbar \omega_{0}+\hbar \Omega\right)^{2}}\left[\delta_{i k}+\omega_{c} \tau\left(\varepsilon_{F}-\hbar \omega_{0}+\hbar \Omega\right) \varepsilon_{i k j}+\right.\right.$ $\left.\omega_{c}{ }^{2} \tau\left(\varepsilon_{F}-\hbar \omega_{0}+\hbar \Omega\right)^{2} h_{i} h_{k}\right]+\frac{\hbar \omega_{0}+\hbar \Omega}{T} S H 6 \frac{\tau\left(\varepsilon_{F}-\hbar \omega_{0}-\hbar \Omega\right)}{1+\omega_{c}{ }^{2} \tau\left(\varepsilon_{F}-\hbar \omega_{0}-\hbar \Omega\right)^{2}}\left[\delta_{i k}+\omega_{c} \tau\left(\varepsilon_{F}-\hbar \omega_{0}-\hbar \Omega\right) \varepsilon_{i k j}+\right.$ $\left.\omega_{c}^{2} \tau\left(\varepsilon_{F}-\hbar \omega_{0}-\hbar \Omega\right)^{2} h_{i} h_{k}\right]-\frac{\hbar \omega_{0}+\hbar \Omega}{T} S H 7 \frac{\tau\left(\varepsilon_{F}+\hbar \omega_{0}+\hbar \Omega\right)}{1+\omega_{c}^{2} \tau\left(\varepsilon_{F}+\hbar \omega_{0}+\hbar \Omega\right)^{2}}\left[\delta_{i k}+\omega_{c} \tau\left(\varepsilon_{F}+\hbar \omega_{0}+\hbar \Omega\right) \varepsilon_{i k j}+\right.$ $\left.\omega_{c}{ }^{2} \tau\left(\varepsilon_{F}+\hbar \omega_{0}+\hbar \Omega\right)^{2} h_{i} h_{k}\right]-\frac{\hbar \omega_{0}-\hbar \Omega}{T} S H 8 \frac{\tau\left(\varepsilon_{F}+\hbar \omega_{0}-\hbar \Omega\right)}{1+\omega_{c}{ }^{2} \tau\left(\varepsilon_{F}+\hbar \omega_{0}-\hbar \Omega\right)^{2}}\left[\delta_{i k}+\omega_{c} \tau\left(\varepsilon_{F}+\hbar \omega_{0}-\hbar \Omega\right) \varepsilon_{i k j}+\right.$ $\left.\left.\omega_{c}^{2} \tau\left(\varepsilon_{F}+\hbar \omega_{0}-\hbar \Omega\right)^{2} h_{i} h_{k}\right]\right\}$

$\gamma_{i j}=-\frac{1}{2} \sigma_{i j} \varepsilon_{F}, \quad \xi_{i j}=\frac{1}{e} \beta_{i j} \varepsilon_{F}^{2}$

Here: $\tau\left(\varepsilon_{F}\right)$ is the momentum relaxation time, $\delta_{i k}$ is the Kronecker delta, $\varepsilon_{i k j}$ being the antisymmetric Levi - Civita tensor; the Latin symbols $i, j, k$ stand for components $x, y, z$ of the Cartesian coordinates.

$$
\begin{gathered}
\Delta_{n, l}=\frac{2 m^{*}}{\hbar^{2}}\left(\varepsilon_{F}+\frac{1}{2}\left(\frac{e E_{1}}{\omega_{c}}\right)^{2}\right)-\frac{2 \omega_{c} m^{*}}{\hbar}\left(N+\frac{n}{2}+\frac{l}{2}+\frac{1}{2}\right) ; x_{1}=\sqrt{\Delta_{n, l}} ; x_{2}=-\sqrt{\Delta_{n, l}} ; y_{1} \\
=\sqrt{\Delta_{n^{\prime} l^{\prime}}} ; y_{2}=-\sqrt{\Delta_{n^{\prime} l^{\prime}}} \\
S H 1=-\frac{x_{1}^{2}}{\sqrt{\Delta_{11} \Delta_{n, l}}}\left(\frac{1}{c_{1}}+\frac{1}{d_{1}}-\frac{e^{2} E_{o}^{4}}{2 m^{2} \Omega^{4}}\left(c_{1}-d_{1}\right)\right)-\frac{x_{2}^{2}}{\sqrt{\Delta_{12} \Delta_{n, l}}}\left(\frac{1}{c_{2}}+\frac{1}{d_{2}}-\frac{e^{2} E_{o}^{4}}{2 m^{2} \Omega^{4}}\left(c_{2}-d_{2}\right)\right) \\
S H 2=\frac{1}{\sqrt{\Delta_{I 1} \Delta_{n^{\prime} l^{\prime}}}}\left[y_{1}\left(y_{1}-C_{1}\right)\left(\frac{1}{C_{1}}-\frac{e^{2} E_{o}^{4}}{2 m^{2} \Omega^{4}} C_{1}\right)+y_{2}\left(y_{2}-C_{2}\right)\left(\frac{1}{C_{2}}-\frac{e^{2} E_{o}^{4}}{2 m^{2} \Omega^{4}} C_{2}\right)\right] \\
+\frac{1}{\sqrt{\Delta_{I 2} \Delta_{n^{\prime} l^{\prime}}}}\left[y_{1}\left(y_{1}-D_{1}\right)\left(\frac{1}{D_{1}}-\frac{e^{2} E_{o}^{4}}{2 m^{2} \Omega^{4}} D_{1}\right)+y_{2}\left(y_{2}-D_{2}\right)\left(\frac{1}{D_{2}}-\frac{e^{2} E_{o}^{4}}{2 m^{2} \Omega^{4}} D_{2}\right)\right]
\end{gathered}
$$

If $\left(c_{1}, d_{1}, c_{2}, d_{2}, \Delta_{11}, \Delta_{12}\right)$ change to $\left(m_{1}, q_{1}, m_{2}, q_{2}, \Delta_{41}, \Delta_{42}\right)$ then $S H 1$ becomes $S H 3$; If $\left(C_{1}, D_{1}, C_{2}, D_{2}, \Delta_{I 1}, \Delta_{I 2}\right)$ change to $\left(M_{1}, Q_{1}, M_{2}, Q_{2}, \Delta_{I V 1}, \Delta_{I V 2}\right)$ then $S H 2$ becomes $S H 4$; 


$$
\begin{aligned}
S H 5=\frac{x_{1}^{2}}{\sqrt{\Delta_{21} \Delta_{n, l}}} & \left(g_{1}+h_{1}\right)+\frac{x_{2}^{2}}{\sqrt{\Delta_{22} \Delta_{n, l}}}\left(g_{2}+h_{2}\right) \\
& +\frac{1}{\sqrt{\Delta_{I I 1} \Delta_{n^{\prime} l^{\prime}}}}\left(G_{1}\left(y_{1}-G_{1}\right) y_{1}+H_{1}\left(y_{1}-H_{1}\right) y_{1}\right) \\
& +\frac{1}{\sqrt{\Delta_{I I 2} \Delta_{n^{\prime} l^{\prime}}}}\left(G_{2}\left(y_{2}-G_{2}\right) y_{2}+H_{2}\left(y_{2}-H_{2}\right) y_{2}\right)
\end{aligned}
$$

$\left(g_{1}, h_{1}, g_{2}, h_{2}, G_{1}, H_{1}, G_{2}, H_{2}, \Delta_{21}, \Delta_{22}, \Delta_{I I 1}, \Delta_{I I 2}\right)$

change to $\left(a_{1}, b_{1}, a_{2}, b_{2}, A_{1}, B_{1}, A_{2}, B_{2}, \Delta_{31}, \Delta_{32}, \Delta_{I I I 1}, \Delta_{I I I 2}\right)$

then $S H 5$ becomes $S H 6$

$$
\left(g_{1}, h_{1}, g_{2}, h_{2}, G_{1}, H_{1}, G_{2}, H_{2}, \Delta_{21}, \Delta_{22}, \Delta_{I I 1}, \Delta_{I I 2}\right)
$$

change to $\left(v_{1}, t_{1}, v_{2}, t_{2}, V_{1}, T_{1}, V_{2}, T_{2}, \Delta_{51}, \Delta_{52}, \Delta_{V 1}, \Delta_{V 2}\right)$

then $S H 5$ becomes $S H 7$

$\left(g_{1}, h_{1}, g_{2}, h_{2}, G_{1}, H_{1}, G_{2}, H_{2}, \Delta_{21}, \Delta_{22}, \Delta_{I I 1}, \Delta_{I I 2}\right)$

change to $\left(z_{1}, w_{1}, z_{2}, w_{2}, Z_{1}, W_{1}, Z_{2}, W_{2}, \Delta_{61}, \Delta_{62}, \Delta_{V I 1}, \Delta_{V I 2}\right)$

then $S H 5$ becomes $S H 8$

$$
\Delta_{11}=x_{1}^{2}-\frac{2 m^{*}}{\hbar}\left(\left(\frac{n^{\prime}-n}{2}+\frac{l^{\prime}-l}{2}\right) \omega_{c}-\omega_{0}\right) ; c_{1}=x_{1}+\sqrt{\Delta_{11}} ; d_{1}=x_{1}-\sqrt{\Delta_{11}} ;
$$

$x_{1}$ change to $x_{2}$ then $\Delta_{11}, c_{1}, d_{1}$ become $\Delta_{12}, c_{2}, d_{2}$

$$
\Delta_{I 1}=y_{1}^{2}+\frac{2 m^{*}}{\hbar}\left(\left(\frac{n^{\prime}-n}{2}+\frac{l^{\prime}-l}{2}\right) \omega_{c}-\omega_{0}\right) ; C_{1}=y_{1}+\sqrt{\Delta_{I 1}} ; D_{1}=y_{1}-\sqrt{\Delta_{I 1}} ;
$$

$y_{1}$ change to $y_{2}$ then $\Delta_{I 1}, C_{1}, D_{1}$ become $\Delta_{I 2}, C_{2}, D_{2}$

$$
\Delta_{21}=x_{1}^{2}-\frac{2 m^{*}}{\hbar}\left(\left(\frac{n^{\prime}-n}{2}+\frac{l^{\prime}-l}{2}\right) \omega_{c}-\omega_{0}+\Omega\right) ; g_{1}=x_{1}+\sqrt{\Delta_{21}} ; h_{1}=x_{1}-\sqrt{\Delta_{21}} \text {; }
$$

$x_{1}$ change to $x_{2}$ then $\Delta_{21}, g_{1}, h_{1}$ become $\Delta_{22}, g_{2}, h_{2}$

$$
\Delta_{I I 1}=y_{1}^{2}+\frac{2 m^{*}}{\hbar}\left(\left(\frac{n^{\prime}-n}{2}+\frac{l^{\prime}-l}{2}\right) \omega_{c}-\omega_{0}+\Omega\right) ; G_{1}=y_{1}+\sqrt{\Delta_{I I 1}} ; H_{1}=y-\sqrt{\Delta_{I I 1}} ;
$$

$y_{1}$ change to $y_{2}$ then $\Delta_{I I 1}, G_{1}, H_{1}$ become $\Delta_{I I 2}, G_{2}, H_{2}$

$$
\Delta_{31}=x_{1}^{2}-\frac{2 m^{*}}{\hbar}\left(\left(\frac{n^{\prime}-n}{2}+\frac{l^{\prime}-l}{2}\right) \omega_{c}-\omega_{0}-\Omega\right) ; a_{1}=x_{1}+\sqrt{\Delta_{31}} ; b_{1}=x_{1}-\sqrt{\Delta_{31}} \text {; }
$$

$x_{1}$ change to $x_{2}$ then $\Delta_{31}, a_{1}, b_{1}$ become $\Delta_{32}, a_{2}, b_{2}$

$$
\Delta_{I I I 1}=y_{1}^{2}+\frac{2 m^{*}}{\hbar}\left(\left(\frac{n^{\prime}-n}{2}+\frac{l^{\prime}-l}{2}\right) \omega_{c}-\omega_{0}-\Omega\right) ; A_{1}=y_{1}+\sqrt{\Delta_{I I I 1}} ; B_{1}=y-\sqrt{\Delta_{I I I 1}} ;
$$

$y_{1}$ change to $y_{2}$ then $\Delta_{I I I 1}, A_{1}, B_{1}$ become $\Delta_{I I I 2}, A_{2}, B_{2}$

$$
\Delta_{41}=x_{1}^{2}-\frac{2 m^{*}}{\hbar}\left(\left(\frac{n^{\prime}-n}{2}+\frac{l^{\prime}-l}{2}\right) \omega_{c}+\omega_{0}\right) ; m_{1}=x_{1}+\sqrt{\Delta_{41}} ; q_{1}=x_{1}-\sqrt{\Delta_{41}} ;
$$


$x_{1}$ change to $x_{2}$ then $\Delta_{41}, m_{1}, q_{1}$ become $\Delta_{42}, m_{2}, q_{2}$

$\Delta_{I V 1}=y_{1}^{2}+\frac{2 m^{*}}{\hbar}\left(\left(\frac{n^{\prime}-n}{2}+\frac{l^{\prime}-l}{2}\right) \omega_{c}+\omega_{0}\right) ; M_{1}=y_{1}+\sqrt{\Delta_{I V 1}} ; Q_{1}=y-\sqrt{\Delta_{I V 1}} ;$

$y_{1}$ change to $y_{2}$ then $\Delta_{V I 1}, M_{1}, Q_{1}$ become $\Delta_{V I 2}, M_{2}, Q_{2}$

$\Delta_{51}=x_{1}^{2}-\frac{2 m^{*}}{\hbar}\left(\left(\frac{n^{\prime}-n}{2}+\frac{l^{\prime}-l}{2}\right) \omega_{c}+\omega_{0}+\Omega\right) ; v_{1}=x_{1}+\sqrt{\Delta_{51}} ; t_{1}=x_{1}-\sqrt{\Delta_{51}} ;$

$x_{1}$ change to $x_{2}$ then $\Delta_{51}, v_{1}, t_{1}$ become $\Delta_{52}, v_{2}, t_{2}$

$\Delta_{V 1}=y_{1}^{2}+\frac{2 m^{*}}{\hbar}\left(\left(\frac{n^{\prime}-n}{2}+\frac{l^{\prime}-l}{2}\right) \omega_{c}+\omega_{0}+\Omega\right) ; V_{1}=y_{1}+\sqrt{\Delta_{V 1}} ; T_{1}=y-\sqrt{\Delta_{V 1}} ;$

$y_{1}$ change to $y_{2}$ then $\Delta_{V 1}, V_{1}, T_{1}$ become $\Delta_{V 2}, V_{2}, T_{2}$

$\Delta_{61}=x_{1}^{2}-\frac{2 m^{*}}{\hbar}\left(\left(\frac{n^{\prime}-n}{2}+\frac{l^{\prime}-l}{2}\right) \omega_{c}+\omega_{0}-\Omega\right) ; z_{1}=x_{1}+\sqrt{\Delta_{61}} ; w_{1}=x_{1}-\sqrt{\Delta_{61}} ;$

$x_{1}$ change to $x_{2}$ then $\Delta_{51}, v_{1}, t_{1}$ become $\Delta_{62}, z_{2}, w_{2}$

$\Delta_{V I 1}=y_{1}^{2}+\frac{2 m^{*}}{\hbar}\left(\left(\frac{n^{\prime}-n}{2}+\frac{l^{\prime}-l}{2}\right) \omega_{c}+\omega_{0}-\Omega\right) ; Z_{1}=y_{1}+\sqrt{\Delta_{V I 1}} ; W_{1}=y-\sqrt{\Delta_{V I 1}} ;$

$y_{1}$ change to $y_{2}$ then $\Delta_{V I 1}, Z_{1}, W_{1}$ become $\Delta_{V I 2}, Z_{2}, W_{2}$

Here: $\kappa, \chi_{0}, \chi_{\infty}, \varepsilon_{F}, L$ and $k_{B}$ are the electric constant, the static dielectric constant, the high frequency dielectric constant, the Fermi level, normalization length and the Boltzmann constant, respectively. From these above expressions, we see that the EC expression in the CQW is more complicated than that in the bulk semiconductor. We also found that the difference in the energy spectrum, the wave function and the presence of electromagnetic waves which lead to this complexity. In the next step, we study quantum wire of GaAs/GaAs:Al to see clearly the dependence mentioned above.

\section{Numerical results and discussion}

In this section, we present detailed numerical calculations of the EC in a CQW subjected to the uniform crossed magnetic and electric fields in the presence of a strong EMW. For the numerical evaluation, we consider the $\mathrm{C} \quad \mathrm{QW}$ of GaAs/GaAs:Al with the parameters[7,8]: $\varepsilon_{F}=50 \mathrm{meV}, \mathrm{m}=$ $0.067 m_{0}\left(m_{0}\right.$ is mass of a free electron), $\tau=10^{-12} \mathrm{~s}, L=10^{-9} \mathrm{~m}$.

Figure 1 shows the dependence of the EC on the magnetic field in two cases: with and without the presence of electromagnetic waves. From the graph, we see that the oscillation appears which is controlled by the ratio of the Fermi energy level and cyclotron energy level. Literally, the appearance of the oscillation is the influence of De Haas-van Alphen effect [20]. Moreover, the effect of electromagnetic waves on the EC is clearly observed. The value of the EC is the same in the domain with strong magnetic field (above $0.125(T)$ ) and it is very different in the $0.1(T)$ to $0.125(T)$ magnetic field domain. In particular, the blue dashed line fluctuates and reaches the resonant point while $\mathrm{B}$ is just under $0.11(T)$ with the negative value of $-8\left(K . m .(T . A)^{\wedge}(-1)\right)$, almost doubles than the red line case (without EMW). In other domains of magnetic $B$, the EC value of both cases are approximately the same and oscillations as the magnetic B increases. 
In figure 2, the EC depends linearly on the temperature with a positive slope coefficient but the value of EC is smaller compared to the bulk semiconductors cases. This result is due to the difference in structure, wave function and energy spectrum of CQWIP in comparison with the bulk semiconductors. Also, the presence of electromagnetic waves influence on the EC is quite remarkable, the EC value is the same in the domain of low temperature and have different values in the region with higher temperatures. This result is consistent with those previously reported by using Boltzmann kinetic equation [1]. However, Boltzmann kinetic equation applies only in high temperature conditions, which is the limitation of the Boltzmann kinetic equation. So, we use quantum equations to overcome the above limitations.

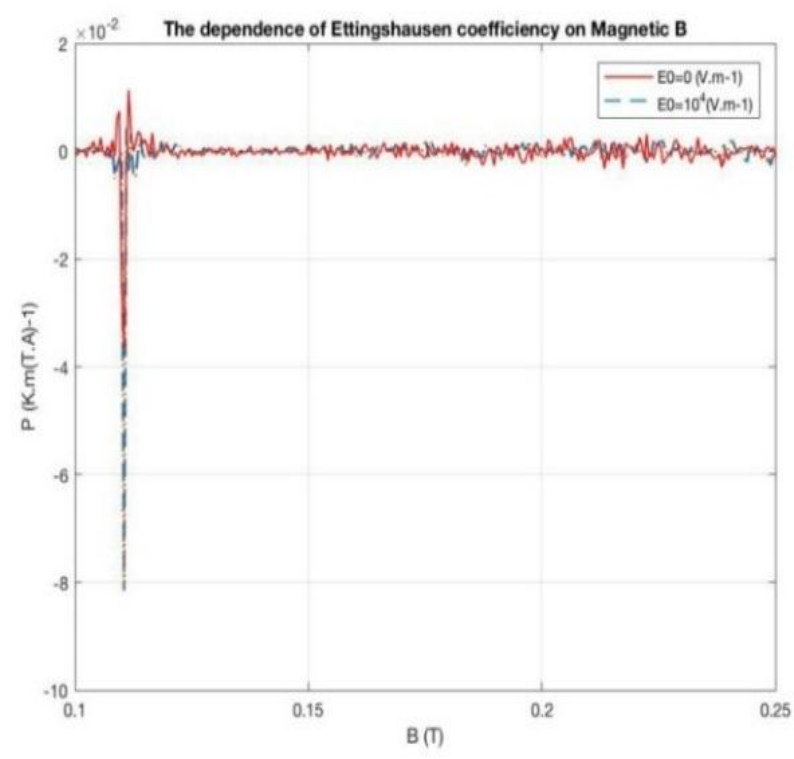

Figure 1. The dependence of Ettingshausen coefficient on magnetic field.

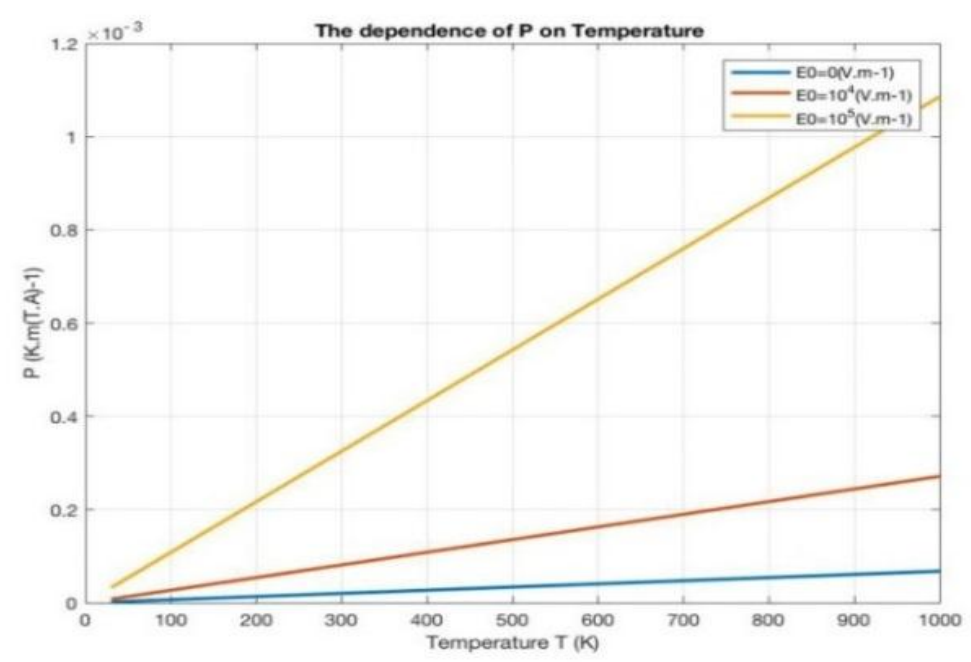

Figure 2.The dependence of Ettingshausen coefficient on temperature $T=30 \mathrm{~K} \div 1000 \mathrm{~K}$. 


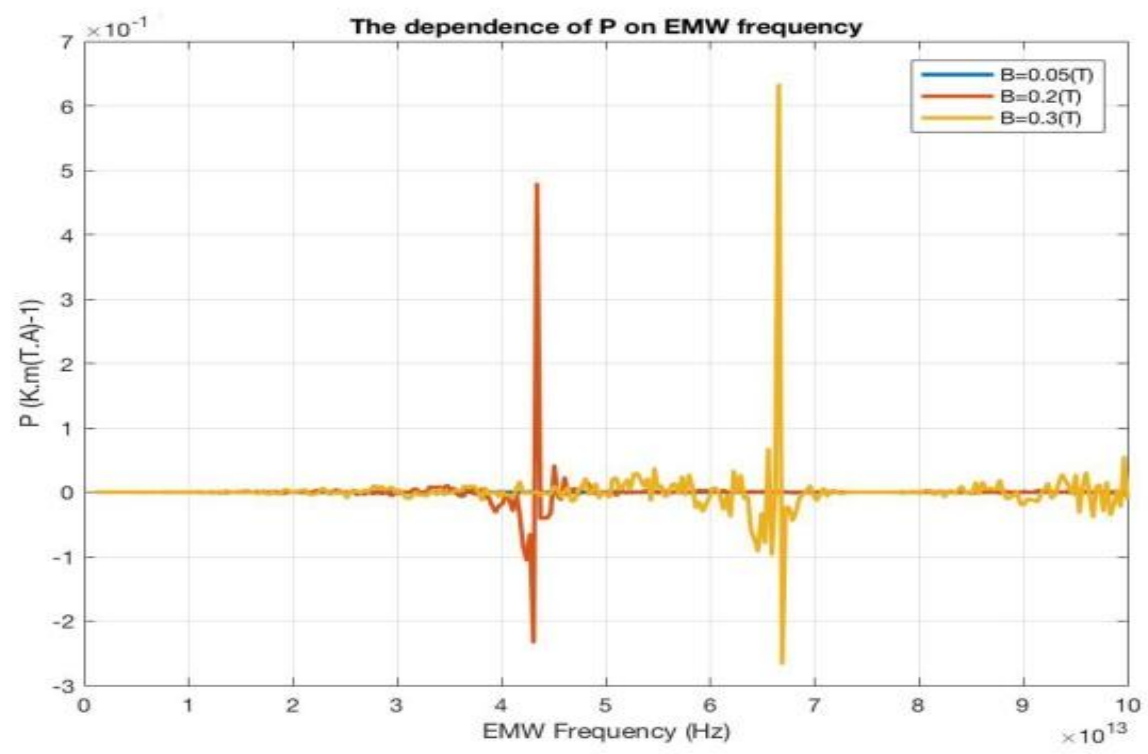

Figure 3. The dependence of Ettingshausen coefficient on the EMW frequency

Figure 3 shows the dependence of EC on the EMW frequency with $\Omega=0 \div 100(\mathrm{THz})$. As can be seen form the graph, the EC oscillates in strong magnetic field condition. In each case of magnetic field, the EC reach a peak with specific value of EMW frequency. When magnetic field value increases, both EC peak value and the peak position of $\Omega$ tends to upwards. This result is similar to the results found in rectangular quantum wire but the depend of EC on $\Omega$ is more significant and the impact of the magnetic field B is different. In this case, at the same point of $\mathrm{B}$, the value of EC is stronger than the EC in the RQW. This can be easily explained by the unalike physical expressions of energy spectrum and single wave function in two different quantum wires.

\section{Conclusion}

In this paper, we have analytically investigated EC in cylindrical quantum wire. The electronoptical phonon interaction is taken into account at both low and high temperatures. We expose the analytical expression of the coefficient EC in CQWIP. The results have been evaluated in GaAs/Al:GaAs CQWIP to see the EC's dependence on electromagnetic wave, temperature, magnetic field.

The results showed that the EC increases linearly with temperature and the EC has a positive value, quite similar to the bulk semiconductors but the $\mathrm{EC}$ is not as strong as in the bulk cases.

When surveying the dependence of EC on magnetic field, we saw the appearance of Shubnikovde Haas oscillations in strong magnetic field domain. Moreover, the impact of EMW is remarkable since the EC value in case of EMW is almost double than the case without EMW.

When we studied the dependence of EC on EMW frequency, we noted that EC oscillates in strong magnetic field condition. The stronger magnetic field is the taller EC oscillation peak is and bigger EMW frequency value at resonant peak is. 


\section{Acknowledgments}

This work was completed with financial support from the National Foundation for Science and Technology Development of Vietnam (Nafosted 103.01-2015.22).

\section{References}

[1] A. Shik, Quantum wells in Physics and Electronics of two-dimensional systems, World Scientific, 1999. https://doi.org/10.1142/3608.

[2] N.Q. Bau, B.D. Hoi, Investigation of the Hall effect in rectangular quantum wells with a perpendicular magnetic field in the presence of high-frequency electromagnetic wave, Int. J. Mod. Phys. B 28 (2014) 1450001. https://doi.org/10.1142/S0217979214500015.

[3] N.Q. Bau, B.D. Hoi, Influence of a strong electromagnetic wave (laser radiation) on the hall effect in quantum wells with a parabolic potential, J. Korean Phys 60 (2012). https://link.springer.com/article/10.3938/jkps.60.59.

[4] N.Q. Bau, T.C. Phong, Parametric resonance of acoustic and optical phonons in a quantum well, J. Korean Phys 42 (2003) 647. https://link.springer.com/article/10.3938/jkps.61.2026.

[5] N.Q. Bau, H.D. Trien, The Nonlinear Absorption Coefficient of Strong Electromagnetic Waves Caused by Electrons Confined in Quantum Wires, J. Korean Phys. Soc. 56 (2010) 120. https://doi.org/10.3938/jkps.56.120.

[6] S.G. Yua, K.W. Kim, M.A. Stroscio, G.J. Iafrate, A. Ballato, Electron interaction with confined acoustic phonons in cylindrical quantum wires via deformation potential, J. Korean Phys 80 (1996) 2815.

[7] M. Meziani, J. Lusakowski, W. Knap, N. Dyakonova, F. Teppe, K.Romanjek, M. Ferrier, R. Clerc, G. Ghibaudo, F. Boeuf, T. Skotnicki, Magnetoresistance characterization of nanometer Si metal-oxide-semiconductor transistors, J. Appl. Phys. 96 (2004) 5761. https://doi.org/10.1063/1.1806991.

[8] Z. Gedik, Z. Tesanovic, Excitation spectrum of a type-II superconductor in a very high magnetic field with randomly pinned vortices, Phys. Rev 52 (1995) 527. https://doi.org/10.1103/PhysRevB.52.527.

[9] P. Zhao, Phonon amplification by absorption of an intense laser field in a quantum well of polar material, Sov.Phys. 49 (1994) 13589. https://doi.org/10.1103/PhysRevB.49.13589.

[10] V.V. Pavlovich, E.M. Epshtein, Nonlinear optical properties of conduction electrons in semiconductors, Sov. Phys. Semicond 11 (1977) 809.

[11] A.A. Pankratov, E.M. Epshtein, Kinetic theory of longitudinal Hall ef- fect in high-frequency electric field , Sov. Phys. Semicond. [Fiz. Tekh. Poluprovodn.] 16 (1982) 1689.

[12] R. Smith, Applied infrared spectroscopy, Semiconductors (Mir, Moskva 1982).

[13] B.V. Paranjape, J.S.Levinger, Theory of the Ettingshausen effect in semiconductors, Phys. Rev 120 (1960) 437 https://doi.org/10.1103/PhysRev.120.437.

[14] V.L. Malevich, E.M. Epshtein, Photostimulated odd magnetoresistance of semiconductors, Sov. Phys. Solid State [Fiz. Tverd. Tela ] 18 (1976) 1286.

[15] V.L. Malevich, E.M. Epshtein, Photostimulated kinetic effects in semiconductors, Sov. Phys 18 (1976) 230.

[16] E.M. Epshtein, Parametric resonance of acoustic and optical phonons in semiconductors, Sov. Phys 120, (1976) 226.

[17] D.T. Hang, D.T. Ha, D.T.T. Thanh, N.Q. Bau, The Ettingshausen coefficient in quantum wells under the influence of laser radiation in the case of electron-optical phonon interaction, PSP 8 (3) (2016) 79-81 http://photonics.pl/PLP/index.php/letters/article/view/8-29.

[18] D.T. Hang, N.V. Nhan, N.Q. Bau, Key Engin. Mater. 783 (2018) 93.

[19] A.D. Levin, G.M. Gusev, O.E. Raichev, A.K. Bakarov, Phys. Rev. B 94, (2016) 115309. https://doi.org/10.1103/PhysRevB.94.115309

[20] A.M. Dyugaev, I.D. Vagner, P. Wyder, On the electron scattering and dephasing by nuclear spins, condmat/0005005 (2000). 